\title{
Design strong anomalous Hall effect via spin canting in antiferromagnetic nodal line materials
}

\author{
Congcong Le $\odot,{ }^{1,2}$ Claudia Felser, ${ }^{1}$ and Yan Sun ${ }^{1, *}$ \\ ${ }^{1}$ Max Planck Institute for Chemical Physics of Solids, 01187 Dresden, Germany \\ ${ }^{2}$ RIKEN Interdisciplinary Theoretical and Mathematical Sciences (iTHEMS), Wako, Saitama 351-0198, Japan
}

(Received 17 May 2021; revised 9 September 2021; accepted 13 September 2021; published 29 September 2021)

The interplay between magnetism and the topological electronic structure provides a large freedom for designing strong anomalous Hall effect (AHE) materials. A nodal line from band inversion is a typical band structure for generating strong AHE. On the other band, in most collinear antiferromagnets (AFMs), the integral of the Berry curvatures on the Brillouin zone is forced to zero by the joint $T O$ symmetry, where $T$ and $O$ are the time reversal and a space group operation, respectively. Even with inverted band structures, such AFM cannot have an AHE. Therefore, the AFM nodal line band structures constructed by spin degenerated bands do not receive much attention in AHE materials. In this work, we illustrate that such a band structure provides a promising starting point for generating strong local and net Berry curvature and, hence, strong intrinsic AHE. In specific AFM compounds of $A \mathrm{MnBi}_{2}(A=\mathrm{Ca}$ and $\mathrm{Yb})$ with an inverted band structure, we observed a strong AHE induced by a weak spin canting from temperature or doping. The anomalous Hall conductivity continues to grow with the canting angle owing to the nodal line in the band structure. Since such spin canting can be adjusted experimentally via doping, it provides another effective strategy to generate and manipulate a strong AHE.

DOI: 10.1103/PhysRevB.104.125145

\section{INTRODUCTION}

In recent years, materials with a strong anomalous Hall effect (AHE) [1] have attracted extensive attention in the fields of materials science and condensed matter physics, which are connected to fundamental topological band structures and potential applications in electronic devices. Since the intrinsic AHE can be understood as the integral of the Berry curvature in the momentum space [2-4], band structures with strong Berry curvature are desired to obtain a strong AHE. Weyl points and nodal lines are two types of promising band structures, which can generate strong local Berry curvatures. Using this guiding principle, strong AHE materials with both large anomalous Hall conductivity (AHC) and anomalous angles were observed in the ferromagnetic Weyl semimetal $\mathrm{Co}_{3} \mathrm{Sn}_{2} \mathrm{~S}_{2}$ [5-7], nodal semimetals of layered ferromagnetic compound $\mathrm{Fe}_{3} \mathrm{GeTe}_{2}[8,9]$, and Heusler compound $\mathrm{Co}_{2} \mathrm{Mn}(\mathrm{Ga} / \mathrm{Al})$ [10-15] et al.

A crucial property of the Berry curvature is that it is odd under a time reversal operator [4]. Hence AHE can exist in a broken time-reversal system in the linear response region. Owing to the broken time reversal symmetry $T$, the ferromagnet can host a nonzero AHE. However, in the collinear antiferromagnets (AFM), despite the broken time reversal

\footnotetext{
*Corresponding author: ysun@cpfs.mpg.de
}

Published by the American Physical Society under the terms of the Creative Commons Attribution 4.0 International license. Further distribution of this work must maintain attribution to the author(s) and the published article's title, journal citation, and DOI. Open access publication funded by the Max Planck Society. operation $T$, there are two types of combined symmetries $T O$ between the time reversal operation $T$ and a unitary space group operation $O$, where $O$ is a fractional translation or inversion symmetry. Then, the combined symmetry $T O$ can also change the sign of the Berry curvature, resulting in the cancellation of the Berry curvature when integrated over the full Brillouin zone (BZ). Therefore, AHE can only exist in a few situations without the combined symmetries $T O$, such as noncollinear AFM in FeMn, $\mathrm{NiS}_{2}$, cubic $\mathrm{Mn}_{3}(\mathrm{Ir} / \mathrm{Pt})$ and hexagonal $\mathrm{Mn}_{3}(\mathrm{Ge} / \mathrm{Sn})$ [16-22], and collinear AFM $\mathrm{Ti}_{2} \mathrm{MnAl}$ and $\mathrm{RuO}_{2}[23,24]$. On the other hand, AMF with combined symmetries $T O$ almost did not attract much attention in AHE materials, even with nodal-line-like special band structures. In addition, based on the effective model, high-temperature quantum AHE can be induced by canted antiferromagnetism [25]. Symmetry and effective model analysis in a two dimensional insulating system suggested the existence of the quantum anomalous Hall phase [25]. However, neither a strong or quantized anomalous Hall effect was observed in realistic materials. In this work, by using first principles, as we will show below, by spin canting, the nodalline-like special band structures in the AFM materials with combined symmetries may generate strong AHE. In addition, the intrinsic contribution is mainly dependent on the electric structures, while the extrinsic effect depends on the details of scattering. Therefore, we mainly focus on the intrinsic contribution.

In this work, we propose an effective strategy for generating strong Berry curvature by spin-canting based on collinear AFM nodal line band structures with combined symmetries $T O$. Figures 1(a)-1(c) show the schematic of AHE in the spin canted AFM order with inverted band structure. At the canting angle $\theta=0$, the bands are doubly degenerate owing to time 

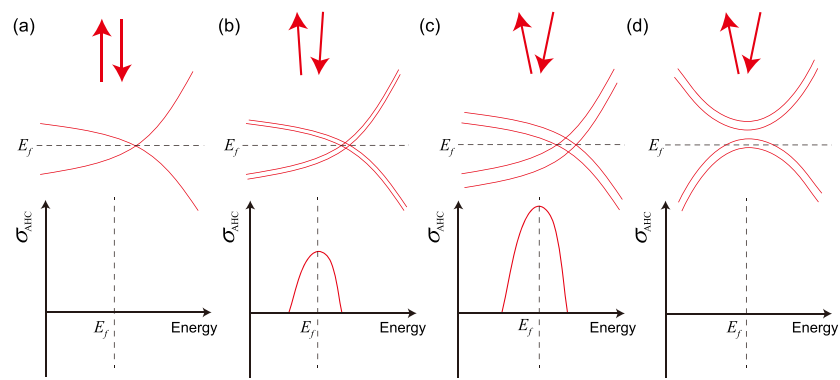

FIG. 1. Schematic of AHE in the spin canted AFM order with (a)-(c) and without (d) band inversion, where $\sigma_{\mathrm{AHC}}$ is the total value of AHC and $E_{f}$ are the AHC and Fermi level, respectively. (a) Canting angle $\theta=0$. (b) Small canting angle $\theta$. (c) Large canting angle $\theta$. (d) AHE in the spin canted AFM order without band inversion at a large canting angle $\theta$.

reversal and inversion symmetries, and hence $\sigma_{\mathrm{AHC}}$ is zero, as shown in Fig. 1(a). When the canting angle $\theta$ is small, the two degenerate bands are split because of the broken time reversal symmetry, suggesting that $\sigma_{\mathrm{AHC}}$ should be nonzero, as shown in Fig. 1(b). Moreover, because the band inversion already exists in spin-degenerated bands, a canting can change the shape of the Berry curvature and AHC. When the canting angle $\theta$ becomes large, the two degenerate bands are split further and $\sigma_{\mathrm{AHC}}$ may become larger, as shown in Fig. 1(c). However, if the canted AFMs cannot host topological band structures, their AHEs are zero, as shown in Fig. 1(d). Based on the above schematic, we perform an $a b$ initio analysis for AHE as the canting angle changes in the spin canting C-type AFM materials $A \mathrm{MnBi}_{2}(A=\mathrm{Ca}$ and $\mathrm{Yb})$ [26-32], where the band inversion leads to a topological nodal ring without considering spin-orbital coupling (SOC). We find that the AHC continues to grow as the canting angle increases. Further, doping electrons can reduce the AHC while doping holes strengthen it.

\section{METHOD AND SYMMETRY ANALYSIS}

\section{A. Method}

Our calculations are performed using density functional theory (DFT) as implemented in the Vienna ab initio simulation package (VASP) code [33-35]. The PerdewBurke-Ernzerhof (PBE) exchange-correlation functional and the projector-augmented-wave (PAW) approach are utilized. Throughout the work, the cutoff energy is set to be $550 \mathrm{eV}$ to expand the wave functions into a plane-wave basis. The Brillouin zone is sampled in the $k$ space within the MonkhorstPack scheme [36] and the $k$ mesh used is $10 \times 10 \times 4$ on the basis of the equilibrium structure. In our calculations, a C-type AFM order along the $c$ axis, a spin canting, and SOC are included. We use parameter "I-CONSTRAINED-M" to constrain the direction of the magnetic moments, and the size can be obtained by self-consistent calculation.

To calculate the AHC, we project the $a b$ initio DFT Bloch wave function into highly symmetric atomic-orbitallike Wannier functions [37] with a diagonal position operator, as implemented in the Vienna ab initio simulation package (VASP) code [33-35]. To obtain precise Wannier functions, we (a)

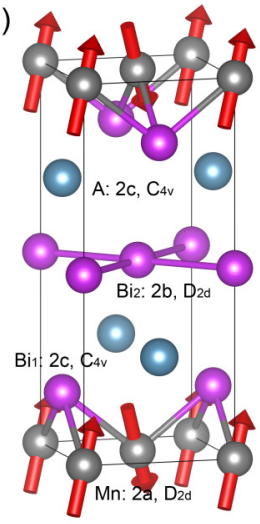

(b)

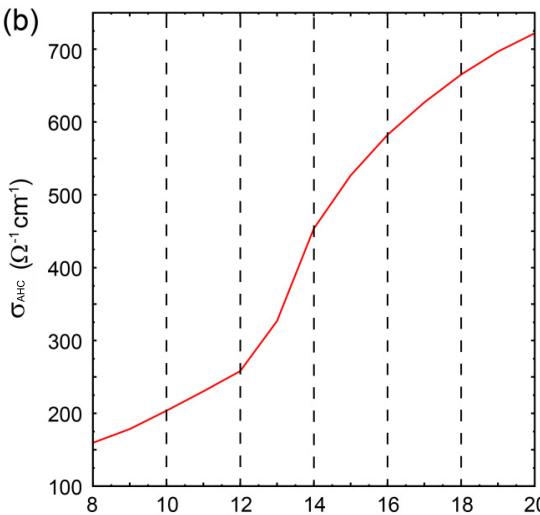

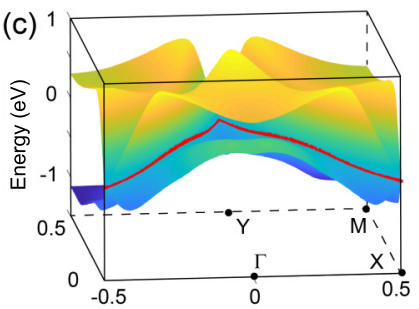

(d)

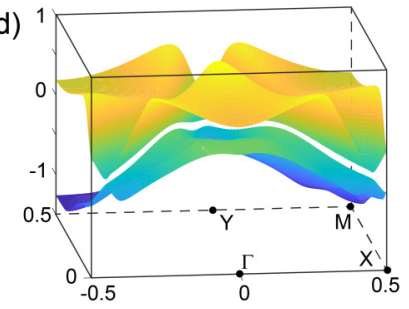

FIG. 2. (a) Crystal structure and C-type AFM order of $A \mathrm{MnBi}_{2}$ with spin canting along the $(1,1,0)$ direction. The Wyckoff positions and corresponding site symmetry groups are provided. (b) Canting angle dependence of the $\mathrm{AHC} \sigma_{\mathrm{AHC}}$ in the $\mathrm{CaMnBi}_{2}$. (c),(d) Band structures in the $k_{z}=0$ plane without and with SOC, where the red line is a nodal line protected by the glide plane symmetry and SOC can break the nodal line into opened gaps. The momentum is measured in units of $2 \pi / a$.

include the outermost $s$ and $d$ orbitals for $\mathrm{Ca}, d$ orbitals for element $\mathrm{Mn}$, and $p$ orbitals for element $\mathrm{Bi}$, which guarantees the full band overlap from $a b$ initio and Wannier functions.

\section{B. Symmetry analysis}

The crystal structure of $A \mathrm{MnBi}_{2}(A=\mathrm{Ca}$ and $\mathrm{Yb})$ with a nonsymmorphic space group $G=P 4 / \mathrm{nmm}$ (No. 129) is shown in Fig. 2(a), where the $\mathrm{MnBi}_{1}$ layers possess an anti-PbO-type atom arrangement and there is a square lattice sheet of $\mathrm{Bi}_{2}$ atoms. $\mathrm{Mn}$ and $\mathrm{Bi}_{2}$ atoms occupy the Wyckoff positions $2 a\{(0,0,0),(1 / 2,1 / 2,0)\}$ and $2 b$ $\{(0,0,1 / 2),(1 / 2,1 / 2,1 / 2)\}$, respectively, which have the same corresponding site symmetry group $D_{2 d}$, whereas $A$ and $\mathrm{Bi}_{1}$ atoms occupy the same Wyckoff positions $2 c$ $\{(1 / 2,0, z),(0,1 / 2,-z)\}$ with the corresponding site symmetry group $C_{4 v}$. In the nonsymmorphic space group $G=$ $P 4 / n m m$, the quotient group $G / L$ is specified by 16 symmetry operations, where $L$ is the translation group with respect to the unit cell. The nonsymmorphic symmetry operations are $\tilde{C}_{2, x}=\left\{C_{2, x} \mid 1 / 2,1 / 2,0\right\}, \tilde{C}_{2, y}=\left\{C_{2, y} \mid 1 / 2,1 / 2,0\right\}$ and $\tilde{M}_{x y}=\left\{M_{x y} \mid 1 / 2,1 / 2,0\right\}$ with respect to the original point at the $\mathrm{Mn}$ site.

Since the $A \mathrm{MnBi}_{2}$ has $\mathrm{AFM}$ order at the $\mathrm{Mn}$ atoms with spins along the $z$ axis, we present the following analysis of magnetic space groups and point groups. Choosing the $\mathrm{Mn}$ site as the origin, the 16 symmetry operations can be specified equivalently as $D_{2 d} \oplus\{I \mid 1 / 2,1 / 2,0\} D_{2 d}$, where $I$ is a space 
inversion and the generators of $D_{2 d}$ are $C_{2, z}, C_{2, x y}$, and $M_{x z}$. First, owing to the spin at the Mn atoms along the $z$ axis, $C_{2, z}$ cannot alter the direction of spin, while $C_{2, x y}$ and $M_{x z}$ flip spin, indicating that $C_{2, x y}$ and $M_{x z}$ are broken by the AFM order at the $\mathrm{Mn}$ atoms. Combined with time reversal symmetry $T$, we obtain the magnetic point group $\overline{4} 2^{\prime} m^{\prime}$ corresponding to the point group $D_{2 d}$, whose generators are $C_{2, z}, T C_{2, x y}$, and $T M_{x z}$. Secondly, since the crystal symmetries in the $\tilde{I} D_{2 d}$ with $\tilde{I}=\{I \mid 1 / 2,1 / 2,0\}$ exchange the Mn sublattice and inversion symmetry $I$ cannot flip spin, $C_{2, x y}$ and $M_{x z}$ are maintained while $C_{2, z}$ is broken. This suggests that the magnetic generators corresponding to $\tilde{I} D_{2 d}$ are $T \tilde{I}, T \tilde{I} C_{2, z}, \tilde{I} C_{2, x y}$, and $\tilde{I} M_{x z}$. Finally, based on the above analysis, we find that the magnetic space group of $A \mathrm{MnBi}_{2}$ with AFM order along the $z$ axis is $P 4^{\prime} / n^{\prime} m^{\prime} m$ with the magnetic point group $4^{\prime} / \mathrm{m}^{\prime} \mathrm{m}^{\prime} m$. Due to $T \tilde{I}$ symmetry, the integral of the Berry curvatures on the BZ is forced to zero.

The existence of spin canting in $\mathrm{AMnBi}_{2}$ leads to an inplane ferromagnetic order, and the magnitude of the canting angle can be experimentally adjusted by doping or temperature [31,32], where they take into account canting angle $10^{\circ}$ to explain experimental results. We consider the same canting direction $(1,1,0)$ as in papers in [31,32], which can break the $T \tilde{I}$ symmetry, indicating that AHE can exist. Then, the corresponding magnetic space group becomes $m^{\prime} m 2^{\prime}$, where the symmetries are $T C_{2, \bar{x} y}, M_{11}, T M_{x y}$, and $E$, which can host a nonzero AHE.

The AHC tensor can be written as

$$
\begin{aligned}
\sigma_{\alpha \beta} & =-\sum_{n} \frac{e^{2}}{\hbar} \int_{\mathrm{BZ}} \frac{d \mathbf{k}}{(2 \pi)^{3}} f_{n}(\mathbf{k}) \boldsymbol{\Omega}_{n, \alpha \beta}(\mathbf{k}), \\
\boldsymbol{\Omega}_{n, \alpha \beta}(\mathbf{k}) & =-2 \operatorname{Im}\left\langle\nabla_{\alpha} u_{n}(\mathbf{k}) \mid \nabla_{\beta} u_{n}(\mathbf{k})\right\rangle,
\end{aligned}
$$

where $\alpha, \beta=x, y, z$ and $n$ is the band index. $f_{n}(\mathbf{k})$ is the Fermi-Dirac distribution, $\left|u_{n}(\mathbf{k})\right\rangle$ is the eigenvalue of the Hamiltonian, and $\boldsymbol{\Omega}_{n, \alpha \beta}(\mathbf{k})$ is the Berry curvature. Under crystal symmetry $g$, the relationship of the Berry curvature $\boldsymbol{\Omega}_{n, \alpha \beta}(\mathbf{k})$ between $\mathbf{k}$ and $g \mathbf{k}$ is given by

$$
\begin{aligned}
\boldsymbol{\Omega}_{n, \alpha \beta}(g \mathbf{k}) & =-2 \operatorname{Im}\left\langle\frac{\partial u_{n}(g \mathbf{k})}{\partial k_{\alpha}} \mid \frac{\partial u_{n}(g \mathbf{k})}{\partial k_{\beta}}\right\rangle \\
& =-2 \sum_{\alpha^{\prime} \beta^{\prime}} \operatorname{Im}\left\langle\frac{\partial(g \mathbf{k})_{\alpha^{\prime}}}{\partial \mathbf{k}_{\alpha}} \frac{\partial u_{n}(g \mathbf{k})}{\partial(g \mathbf{k})_{\alpha^{\prime}}} \mid \frac{\partial(g \mathbf{k})_{\beta^{\prime}}}{\partial \mathbf{k}_{\beta}} \frac{\partial u_{n}(g \mathbf{k})}{\partial(g \mathbf{k})_{\beta^{\prime}}}\right\rangle \\
& =\sum_{\alpha^{\prime} \beta^{\prime}} \frac{\partial(g \mathbf{k})_{\alpha^{\prime}}}{\partial \mathbf{k}_{\alpha}} \frac{\partial(g \mathbf{k})_{\beta^{\prime}}}{\partial \mathbf{k}_{\beta}} \boldsymbol{\Omega}_{n, \alpha^{\prime} \beta^{\prime}}(\mathbf{k}) \\
\boldsymbol{\Omega}_{n, \alpha^{\prime} \beta^{\prime}}(\mathbf{k}) & =-2 \operatorname{Im}\left\langle\frac{\partial u_{n}(\mathbf{k})}{\partial \mathbf{k}_{\alpha^{\prime}}} \mid \frac{\partial u_{n}(\mathbf{k})}{\partial \mathbf{k}_{\beta^{\prime}}}\right\rangle
\end{aligned}
$$

Here, $\frac{\partial(g \mathbf{k})_{\alpha^{\prime}}}{\partial \mathbf{k}_{\alpha}} \frac{\partial(g \mathbf{k})_{\beta^{\prime}}}{\partial \mathbf{k}_{\beta}}$ is a constant because $g \mathbf{k}$ is a linear function of $\mathbf{k}$. Hence $\frac{\partial(g \mathbf{k})_{\alpha^{\prime}}}{\partial \mathbf{k}_{\alpha}} \frac{\partial(g \mathbf{k})_{\beta^{\prime}}}{\partial \mathbf{k}_{\beta}}$ is unchanged when we make a substitution $g \mathbf{k} \rightarrow \mathbf{k}$. It is clear that the AHC is invariant under the inversion symmetry $I$, namely $\boldsymbol{\Omega}_{n, \alpha \beta}^{\gamma}(\mathbf{k})=\boldsymbol{\Omega}_{n, \alpha \beta}^{\gamma}(I \mathbf{k})$. Then, we consider the constraint of the magnetic space group $m^{\prime} m 2^{\prime}$ on the AHC, where considering the mirror symmetry $M_{11}:\left(\mathbf{k}_{x}, \mathbf{k}_{y}, \mathbf{k}_{z}\right) \rightarrow\left(-\mathbf{k}_{y},-\mathbf{k}_{x}, \mathbf{k}_{z}\right)$ is sufficient. Under the mirror symmetry $M_{11}$, the transformation of the Berry curvature $\boldsymbol{\Omega}_{n, \alpha \beta}^{\gamma}(\mathbf{k})$ can be given by

$$
\Omega_{x y}^{z}(\mathbf{k})=-\Omega_{x y}^{z}\left(M_{11} \mathbf{k}\right), \quad \Omega_{x z}^{y}(\mathbf{k})=-\Omega_{y z}^{x}\left(M_{11} \mathbf{k}\right) .
$$

Hence, under the constraint of the magnetic space group $m^{\prime} m 2^{\prime}$, the shape of the AHC tensors is

$$
\left(\begin{array}{ccc}
0 & 0 & \sigma_{x z} \\
0 & 0 & -\sigma_{x z} \\
-\sigma_{x z} & \sigma_{x z} & 0
\end{array}\right) .
$$

\section{III. $A b$ initio ANALYSIS OF ANOMALOUS TRANSPORTS}

Figures 2(c) and 2(d) show the band structures without and with SOC in the $k_{z}=0$ plane, where half of the BZ $(-0.5<$ $k_{x}<0.5$ and $\left.0<k_{y}<0.5\right)$ is shown for the convenience of locating the nodal line. There is a band inversion from the intercalated $\mathrm{Bi}_{2} p_{x / y}$ orbitals, which leads to quasi-twodimensional band crossing. When the SOC is ignored, the crossing bands can be referred to as $K$ and $K+Q$ bands. Similar to the symmetry analysis of iron superconductors [38], the $K$ and $K+Q$ bands are the different eigenvalues of the glide plane symmetry [30], indicating that the nodal ring from $\mathrm{Bi}_{2} p_{x / y}$ band crossing is robust, as shown in Fig. 2(c). When the on-site SOC is included, the spin-flip term $\left\langle p_{x}, \sigma\left|H_{\text {soc }}\right| p_{y}, \bar{\sigma}\right\rangle$ with the spin index $\sigma$ can be nonzero [30]. Hence the crossing bands can hybridize each other and the nodal line can be gapped, as shown in Fig. 2(d). Next, we will calculate $\mathrm{AHC}$ and consider how the nodal line contributes to AHC.

We calculated the AHC tensor of $\mathrm{CaMnBi}_{2}$ with the Fermi level lying at the charge neutral points. Figure 2(b) shows the canting angle dependence of the AHC $\sigma_{\mathrm{AHC}}$ with spin canting along the $(1,1,0)$ direction, where $\sigma_{\mathrm{AHC}}=\sqrt{\sigma_{x z}^{2}+\sigma_{y z}^{2}}=$ $\sqrt{2} \sigma_{x z}$ owing to $\sigma_{y z}=-\sigma_{x z}$. At a canting angle of $8^{\circ}$, the AHC $\sigma_{\mathrm{AHC}}$ has a small value of $159.50 \Omega^{-1} \mathrm{~cm}^{-1}$, whereas it has a large value of $721.90 \Omega^{-1} \mathrm{~cm}^{-1}$ at a canting angle of $20^{\circ}$. As the canting angle increases from $8^{\circ}$ to $20^{\circ}$, the AHC $\sigma_{\mathrm{AHC}}$ continues to grow and is not saturated even if the canting angle reaches $20^{\circ}$. In addition, between $12^{\circ}$ and $14^{\circ}$, the rate of increase of the $\mathrm{AHC}$ is larger. To explain the variation of the AHC with the canting angle, we consider three $\left(8^{\circ}, 13^{\circ}\right.$, and $18^{\circ}$ ) canting angles as examples to calculate band structures and local momentum distribution of the Berry curvature in the $\mathrm{BZ}$ with spin canted C-type AFM states, where the magnitude of the magnetic moments in the $x y$ plane $(z$ direction) are $0.53 \mu_{B}\left(3.81 \mu_{B}\right), 0.99 \mu_{B}\left(3.74 \mu_{B}\right)$, and $1.31 \mu_{B}\left(3.63 \mu_{B}\right)$, respectively.

First, Fig. 3(a) shows band structures at a canting angle of $8^{\circ}$. Near the Fermi level, the valence and conduction bands are mainly attributed to the $p$ orbitals of the intercalated $\mathrm{Bi}_{2}$ atoms, similar to band structures without a canting angle [32]. The bands are split into spin up and spin down owing to the ferromagnetic order in the $x y$ plane. The small hole pockets around the $\Gamma$ point are dominated by the intercalated $\mathrm{Bi}_{2}-p_{z}$ (blue) orbital, which is remarkably split because of hybridizing with the Mn $d$ orbitals. The electronic pockets from the nodal line originate mainly from the intercalated $\mathrm{Bi}_{2}-p_{x / y}$ (green) orbitals, which are less sensitive to the ferromagnetic order in the $x y$ plane and are hardly split. Figure 3(d) shows the local momentum distribution of the Berry curvature in 
$8^{\circ}$

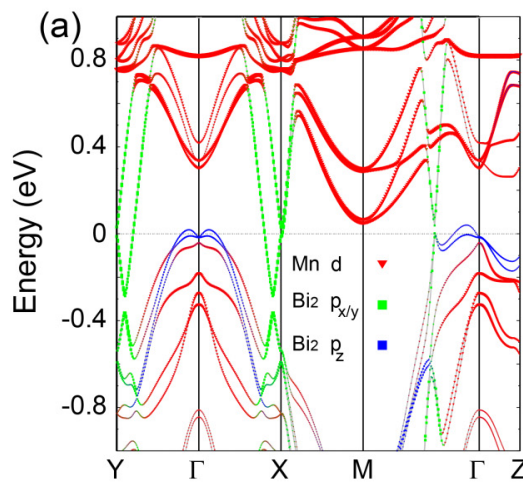

$13^{\circ}$

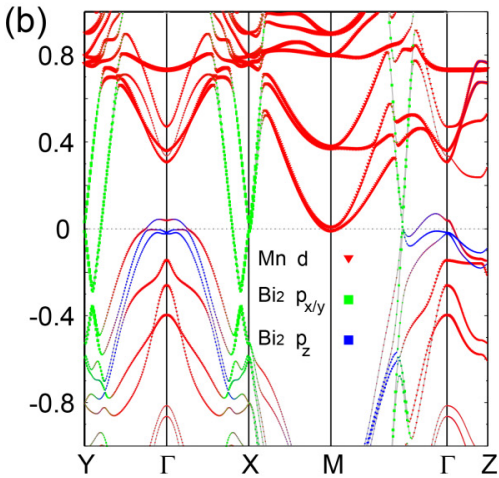

$18^{\circ}$

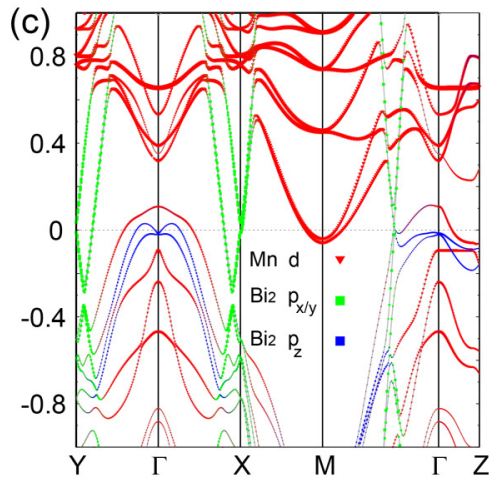

(d) Nodal line
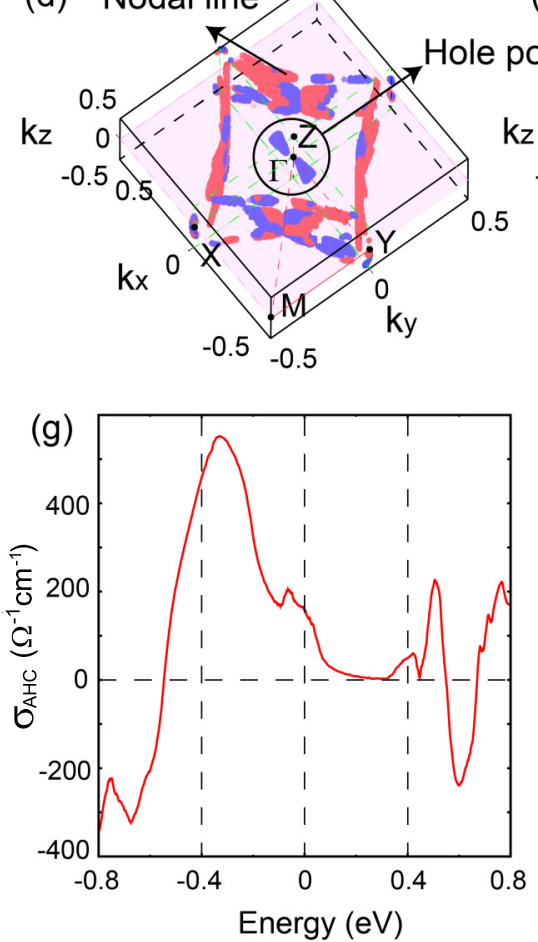

(e)

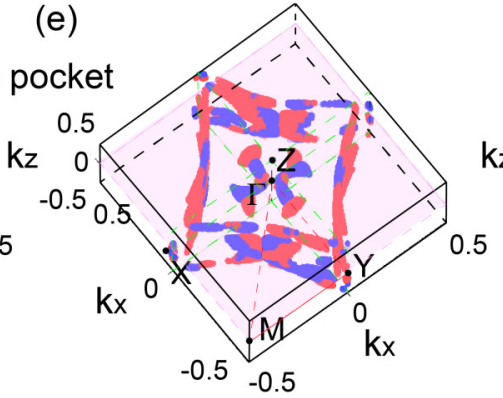

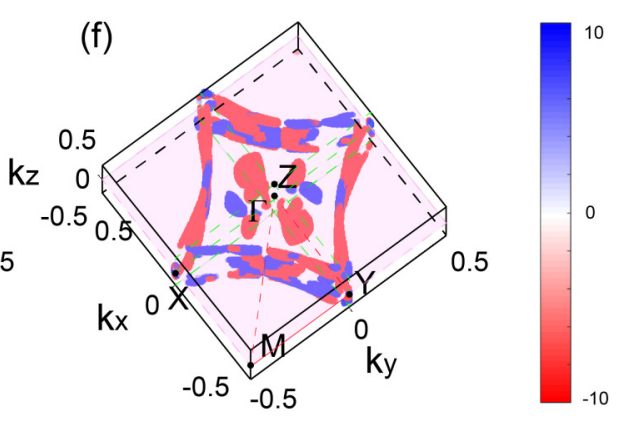
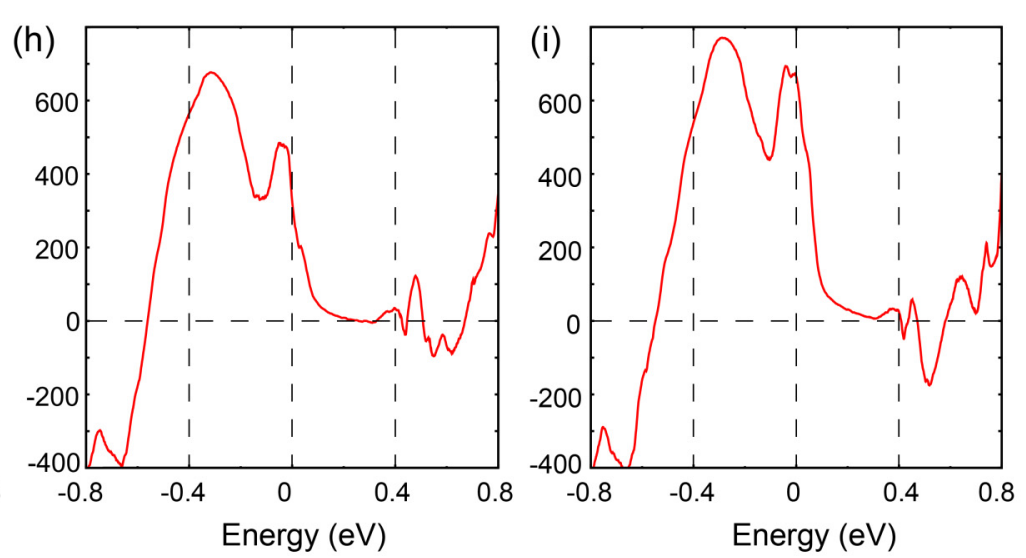

FIG. 3. (a)-(c) Band structures with spin canted C-type AFM states at canting angles of $8^{\circ}, 13^{\circ}$, and $18^{\circ}$, respectively. The orbital characters of the bands are represented by different colors. Panels (d)-(f) show the local momentum distribution of the Berry curvature in the BZ $\sigma_{\text {total }}$ at canting angles of $8^{\circ}, 13^{\circ}$, and $18^{\circ}$, respectively. (g)-(i) Energy-dependent $\mathrm{AHC} \sigma_{\mathrm{AHC}}$ at canting angles of $8^{\circ}, 13^{\circ}$, and $18^{\circ}$, respectively.

the BZ at $8^{\circ}$ canting angle. Around the $\Gamma$ point, the Berry curvatures are mainly contributed by hole pockets with a small volume and positive. The Berry curvatures near the nodal line dominated by the electronic pockets are negative and large volumes. Since the value of AHC $\sigma_{\mathrm{AHC}}$ is $159.50 \Omega^{-1} \mathrm{~cm}^{-1}$, the integral of them gives a negative net Berry curvature, namely, a positive AHC. The nodal line has dispersion in the BZ, which can result in electronic pockets at the Fermi level. Hence the nodal line is mainly responsible for nonzero AHC.

Second, when the canting angle is increased to $13^{\circ}$, the splits of the band structures become larger as the magnitude of the ferromagnetic order in the $x y$ plane increases. Then, the electronic pockets from the nodal line have more splits, which may strengthen AHC. The Mn $d$-orbital bands can also attribute a hole pocket near the $\Gamma$ point, which can result in a positive Berry curvature, as shown in Figs. 3(b) and 3(e). Hence the AHC corresponding to the canting angle of $13^{\circ}$ exceeds that of $8^{\circ}$, as shown in Fig. 2(b). Finally, when the canting angle is further increased to $18^{\circ}$, a large hole pocket near the $\Gamma$ point is completely contributed by the Mn$d$ orbitals owing to the large band splits induced by strong ferromagnetism. The large hole pocket can cause a large and negative Berry curvature, indicating that the $\mathrm{AHC}$ at a canting angle of $18^{\circ}$ increases, as shown in Figs. 3(c) and 3(f).

Here, we summarize the analysis above. We find that the ferromagnetism in the $x y$ plane increases with the canting angle, which can cause greater splits in the band structures. Then, the hole pocket contributed by the Mn $d$ orbitals will become larger, and can cause large and negative Berry curvature. Moreover, the electronic pockets from the nodal line mainly contribute to the AHC, indicating that it is responsible for nonzero AHC. As the ferromagnetism increases, the 

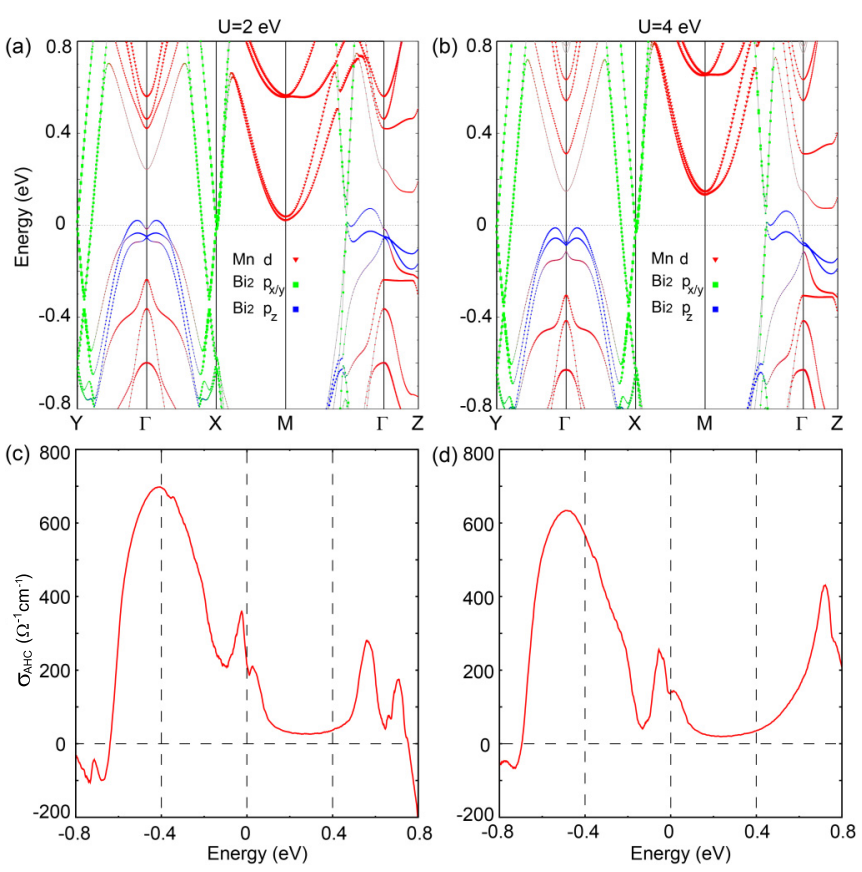

FIG. 4. Band structures and the corresponding energy-dependent AHC tensor $\sigma_{\mathrm{AHC}}$ when the effective on-site Coulomb $U$ are (a),(c) $2 \mathrm{eV}$ and (b),(d) $4 \mathrm{eV}$ for the Mn $d$ orbitals at a canting angle of $18^{\circ}$ in the GGA $+U$ calculation.

electronic pockets split further, which can strengthen the AHC. Therefore, in the range of canting angles we consider, the AHC continues to grow as the canting angle increases.

To further explore the effect of hole and electron doping on the AHC $\sigma_{\mathrm{AHC}}$, Figs. 3(d)-3(f) show the energy-dependent AHC $\sigma_{\mathrm{AHC}}$ calculated from the Berry curvature at canting angles of $8^{\circ}, 13^{\circ}$, and $18^{\circ}$. When the Fermi level is at the charge neutral points, the AHC $\sigma_{\mathrm{AHC}}$ are 110,230 , and $470 \Omega^{-1} \mathrm{~cm}^{-1}$, respectively, indicating continuous growth of the $\mathrm{AHC}$ as the canting angle increases. At canting angles of $13^{\circ}$ and $18^{\circ}$, a peak in $\sigma_{\mathrm{AHC}}$ appears around the Fermi level, as shown in Figs. 3(e) and 3(f). In addition, in three cases, near Fermi level doping electrons can reduce AHC, while doping holes strengthen it.

In order to consider the effect of the correlation effect on AHC in the canted C-type AFM order, Fig. 4 shows band structures and $\mathrm{AHC}$ when the effective on-site Coulomb $U$ are $2 \mathrm{eV}$ and $4 \mathrm{eV}$ for the $\mathrm{Mn} d$ orbitals at a canting angle of $18^{\circ}$ in the GGA $+U$ calculation. The gap between the Mn $d$ orbitals (red) increases as $U$ increases, while the band structures attributed by intercalated $\mathrm{Bi}_{2} p$ orbitals (green and blue) remain unchanged, as shown in Figs. 4(a) and 4(b). Compared to the band structures without $U$, the $d$-orbital bands with $U$ stay away from the Fermi level, and the bands at the Fermi level are mainly attributed to the $\mathrm{Bi}_{2} p$ orbitals. Figures 4(c) and 4(d) show the energy-dependent AHC tensor $\sigma_{\mathrm{AHC}}$ when $U$ are $2 \mathrm{eV}$ and $4 \mathrm{eV}$ for the $\mathrm{Mn} d$ orbitals at a canting angle of $18^{\circ}$, and the AHC tensors $\sigma_{\mathrm{AHC}}$ with $U$ are small compared to those without $U$, indicating that the correlation effect can suppress AHC.

Since spin canting exists in $\mathrm{YbMnBi}_{2}$, we calculate the AHC tensors and band structures of $\mathrm{YbMnBi}_{2}$ at different canting angles. Similar to $\mathrm{CaMnBi}_{2}$, the ferromagnetism in the $x y$ plane increases with the canting angle, and the hole pocket at the $\Gamma$ point contributed by the Mn $d$ orbitals becomes larger, resulting in large and negative Berry curvature. Therefore, the AHC $\sigma_{\mathrm{AHC}}$ in the $\mathrm{YbMnBi}_{2}$ also increases as the canting angle increases. The energy-dependent AHC $\sigma_{\mathrm{AHC}}$ is also calculated at different canting angles, and doping electrons is found to suppress $\mathrm{AHC}$ while doping holes strengthens it.

\section{CONCLUSION}

In summary, the AHE in the layered ternary material $A \mathrm{MnBi}_{2}(A=\mathrm{Ca}$ and $\mathrm{Yb})$ with spin canted C-type antiferromagnetic order can be studied based on first-principles calculations and symmetry analysis. Considering the canting along the $(1,1,0)$ direction, the magnetic space group is $m^{\prime} m 2^{\prime}$, and only $\sigma_{x z / y z}$ in the AHC tensors is nonzero because of mirror symmetry. We find that the AHC $\sigma_{\mathrm{AHC}}$ grows continuously with the canting angle increasing, and doping electrons can reduce AHC while doping holes strengthens it. The correlation effect from the Mn $d$ orbitals is considered, and it can suppress AHC. This work provides an effective design strategy and corresponding materials for obtaining a strong AHE.

\section{ACKNOWLEDGMENTS}

We thank Y. Pan for helpful discussions. This work was financially supported by the ERC Advanced Grant No. 291472 "Idea Heusler," ERC Advanced Grant No. 742068 "TOPMAT," SKYTOP with Grant No. 824123, ASPIN with Grant No. 766566, and DAAD Grant No. 57559136. Some of our calculations were carried out on the Cobra cluster of MPCDF, Max Planck society.
[1] N. Nagaosa, J. Sinova, S. Onoda, A. H. MacDonald, and N. P. Ong, Rev. Mod. Phys. 82, 1539 (2010).

[2] Z. Fang, N. Nagaosa, K. S. Takahashi, A. Asamitsu, R. Mathieu, T. Ogasawara, H. Yamada, M. Kawasaki, Y. Tokura, and K. Terakura, Science 302, 92 (2003).

[3] F. D. M. Haldane, Phys. Rev. Lett. 93, 206602 (2004).

[4] D. Xiao, M. C. Chang, and Q. Niu, Berry phase effects on electronic properties, Rev. Mod. Phys. 82, 1959 (2010).
[5] E. Liu, Y. Sun, N. Kumar, L. Muechler, A. Sun, L. Jiao, S.-Y. Yang, D. Liu, A. Liang, Q. Xu, J. Kroder, V. Su, H. Borrmann, C. Shekhar, Z. Wang, C. Xi, W. Wang, W. Schnelle, S. Wirth, Y. Chen, S. T. B. Goennenwein, and C. Felser, Nat. Phys. 14, 1125 (2018).

[6] Q. Wang, Y. Xu, R. Lou, Z. Liu, M. Li, Y. Huang, D. Shen, H. Weng, S. Wang, and H. Lei, Nat. Commun. 9, 3681 (2018).

[7] Q. Xu, E. Liu, W. Shi, L. Muechler, J. Gayles, C. Felser, and Y. Sun, Phys. Rev. B 97, 235416 (2018). 
[8] C. Tan, J. Lee, S.-G. Jung, T. Park, S. Albarakati, J. Partridge, M. R. Field, D. G. McCulloch, L. Wang, and C. Lee, Nat. Commun. 9, 1554 (2018).

[9] K. Kim, J. Seo, E. Lee, K.-T. Ko, B. S. Kim, B. G. Jang, J. M. Ok, J. Lee, Y. J. Jo, W. Kang, J. H. Shim, C. Kim, H. W. Yeom, B. I. Min, B.-J. Yang, and J. S. Kim, Nat. Mater. 17, 794 (2018).

[10] S. N. Guin, K. Manna, J. Noky, S. J. Watzman, C. Fu, N. Kumar, W. Schnelle, C. Shekhar, Y. Sun, J. Gooth, and C. Felser, NPG Asia Mater. 11, 16 (2019).

[11] P. Li, J. Koo, W. Ning, J. Li, L. Miao, L. Min, Y. Zhu, Y. Wang, N. Alem, C.-X. Liu, Z. Mao, and B. Yan, Nat. Commun. 11, 3476 (2020).

[12] J. Kubler and C. Felser, Europhys. Lett. 114, 47005 (2016).

[13] J. Noky, Q. Xu, C. Felser, and Y. Sun, Phys. Rev. B 99, 165117 (2019).

[14] K. Manna, Y. Sun, L. Muechler, J. Kubler, and C. Felser, Nat. Rev. Mater. 3, 244 (2018).

[15] K. Manna, L. Muechler, T.-H. Kao, R. Stinshoff, Y. Zhang, J. Gooth, N. Kumar, G. Kreiner, K. Koepernik, R. Car, J. Kubler, G. H. Fecher, C. Shekhar, Y. Sun, and C. Felser, Phys. Rev. X 8, 041045 (2018).

[16] R. Shindou and N. Nagaosa, Phys. Rev. Lett. 87, 116801 (2001).

[17] H. Chen, Q. Niu, and A. H. MacDonald, Phys. Rev. Lett. 112, 017205 (2014).

[18] J. Kubler and C. Felser, Europhys. Lett. 108, 67001 (2014).

[19] S. Nakatsuji, N. Kiyohara, and T. Higo, Nature (London) 527, 212 (2015).

[20] A. K. Nayak, J. E. Fischer, Y. Sun, B. Yan, J. Karel, A. C. Komarek, C. Shekhar, N. Kumar, W. Schnelle, J. Kubler, C. Felser, and S. S. P. Parkin, Sci. Adv. 2, e1501870 (2016).

[21] Y. Zhang, Y. Sun, H. Yang, J. zelezny, S. P. P. Parkin, C. Felser, and B. Yan, Phys. Rev. B 95, 075128 (2017).

[22] R. D. dos Reis, M. Ghorbani Zavareh, M. O. Ajeesh, L. O. Kutelak, A. S. Sukhanov, S. Singh, J. Noky, Y. Sun, J. E. Fischer, K. Manna, C. Felser, and M. Nicklas, Phys. Rev. Materials 4, 051401(R) (2020).
[23] W. Shi, L. Muechler, K. Manna, Y. Zhang, K. Koepernik, R. Car, J. van den Brink, C. Felser, and Y. Sun, Phys. Rev. B 97, 060406(R) (2018).

[24] Z. Feng, X. Zhou, L. mejkal, L. Wu, Z. Zhu, H. Guo, R. Gonzalez-Hernandez, X. Wang, H. Yan, P. Qin, X. Zhang, H. Wu, H. Chen, C. Jiang, M. Coey, J. Sinova, T. Jungwirth, and Z. Liu, arXiv:2002.08712.

[25] X. Li, A. H. MacDonald, and H. Chen, arXiv:1902.10650.

[26] J. B. He, D. M. Wang, and G. F. Chen, Appl. Phys. Lett. 100, 112405 (2012).

[27] A. Wang, D. Graf, L. Wu, K. Wang, E. Bozin, Y. Zhu, and C. Petrovic, Phys. Rev. B 94, 125118 (2016).

[28] A. Zhang, C. Liu, C. Yi, G. Zhao, T. L. Xia, J. Ji, Y. Shi, R. Yu, X. Wang, C. Chen, and Q. Zhang, Nat. Commun. 7, 13833 (2016).

[29] Z. Qiu, C. Le, Y. Dai, B. Xu, J. B. He, R. Yang, G. Chen, J. Hu, and X. Qiu, Phys. Rev. B 98, 115151 (2018).

[30] Z. Qiu, C. Le, Z. Liao, B. Xu, R. Yang, J. Hu, Y. Dai, and X. Qiu, Phys. Rev. B 100, 125136 (2019).

[31] S. Borisenko, D. Evtushinsky, Q. Gibson, A. Yaresko, K. Koepernik, T. Kim, M. Ali, J. van den Brink, M. Hoesch, A. Fedorov, E. Haubold, Y. Kushnirenko, I. Soldatov, R. Schafer, and R. J. Cava, Nat. Commun. 10, 3424 (2019).

[32] R. Yang, M. Corasaniti, C. C. Le, Z. Y. Liao, A. F. Wang, Q. Du, C. Petrovic, X. G. Qiu, J. P. Hu, and L. Degiorgi, Phys. Rev. Lett. 124, 137201 (2020).

[33] G. Kresse and J. Hafner, Phys. Rev. B 47, 558 (1993).

[34] G. Kresse and J. Furthmuller, Comput. Mater. Sci. 6, 15 (1996).

[35] G. Kresse and J. Furthmuller, Phys. Rev. B 54, 11169 (1996).

[36] H. J. Monkhorst and J. Pack, Phys. Rev. B 13, 5188 (1976).

[37] J. R. Yates, X. Wang, D. Vanderbilt, and I. Souza, Phys. Rev. B 75, 195121 (2007).

[38] X. Wu, S. Qin, Y. Liang, H. Fan, and J. Hu, Phys. Rev. B 93, 115129 (2016). 